\title{
CUIDADO DE ENFERMAGEM NA APAE: NECESSIDADES DA EQUIPE MULTIPROFISSIONAL
}

\author{
Nursing care at the APAE: needs of the multiprofessional team
}

Luana Eugênio Rosso ${ }^{1}$, Ana Regina da Silva Losso ${ }^{1}$

${ }^{1}$ Universidade do Extremo Sul Catarinense (UNESC).

\section{Endereço para correspondência:}

Luana Eugênio Rosso

Rua Lourenço Zanette, 335, bloco 11 Apt. 402, Residencial Padova 339, Santo Antônio, Criciúma - SC.

CEP: 88809-470

Email: luana.rosso@outlook.com 


\title{
Resumo
}

Estudo com o objetivo de identificar os desafios enfrentados pela equipe multiprofissional da Associação de Pais e Amigos dos Excepcionais (APAE) sem a inclusão de um profissional enfermeiro na instituição. Pesquisa de abordagem qualitativa, descritiva e exploratória de campo. O estudo foi desenvolvido em um município do Extremo Sul de Santa Catarina. Aplicou-se questionário semiestruturado com 14 profissionais da equipe multiprofissional da instituição. Os dados foram analisados a partir da categorização, ordenação e classificação das informações coletadas. O principal desafio apontado foi o enfrentamento de situações clínicas da área da saúde por profissionais não habilitados para tal função, devido à ausência na equipe do profissional Enfermeiro. Como resultado da temática sugere-se a incorporação do enfermeiro na equipe multiprofissional, pois proporcionaria maior segurança, eficácia e qualidade na assistência prestada, possibilitando melhor desenvolvimento e qualidade de vida ao aluno da APAE.

Palavras-chave: Enfermagem; APAE; Equipe Multidisciplinar; Trabalho.

\begin{abstract}
Study in order to identify the challenges faced by the multidisciplinary team of APAE without the inclusion of a professional nurse in the institution. qualitative research, descriptive, exploratory field. The study was developed in a city of the extreme south of Santa Catarina. Semi-structured questionnaire was applied to 14 professionals of the multidisciplinary team of the institution. Data were analyzed from the categorization, sorting and classification of the information collected. The main challenge was appointed coping with medical conditions of health professionals by not qualified for this role due to the absence in the nurse professional. As a result of the theme is suggested the incorporation of the nurse in the multidisciplinary team as provide greater safety, efficacy and quality of care provided, enabling better development and quality of life to the students of APAE.
\end{abstract}

Keywords: Nursing; APAE; Multidisciplinary Team; Work. 
Pelas estimativas da Organização Mundial da Saúde (OMS), em todo o mundo existem pelo menos um bilhão de pessoas com algum tipo de deficiência, representadas por qualquer perda ou anormalidade da estrutura ou função psicológica, fisiológica ou anatômica do indivíduo. As deficiências estão relacionadas não só à condição individual e intrínseca da pessoa, mas também à sua relação com o ambiente social, com os padrões socialmente aceitos como referenciais e representativos do que seria a normalidade. Portanto, para atender ao portador de deficiência, faz-se necessário uma equipe multiprofissional que tenha como pressuposto a integralidade do cuidado e o desenvolvimento das potencialidades dessa população'.

Face à relevância das deficiências na atualidade e considerando-se que na maioria das instituições não existe a inclusão de profissionais da enfermagem para a assistência aos alunos, família e própria equipe; considerando-se a importância da inclusão da pessoa com deficiência e família na sociedade; considerando-se também a vivência da própria pesquisadora com esta realidade na família, escolheu-se a temática proposta.

Essa pesquisa surgiu do interesse da acadêmica do curso de enfermagem pela área de saúde mental, necessidade de expandir a área de atuação do enfermeiro, pela demanda dos usuários da Associação de Pais e Amigos dos Excepcionais (APAE) na região oeste de Santa Catarina e por acreditar que se põem outros cenários de práticas e de contribuir na melhoria da qualidade de vida das pessoas com necessidades especiais.

Em uma instituição de educação especial, a exemplo da APAE, encontramos pessoas com rebaixamento intelectual, transtornos físicos e mentais associados e situações clínicas com características próprias e singulares, o que confere a necessidade de prestação da assistência feita por uma equipe multiprofissional qualificada e competente para atender às necessidades de saúde apresentadas por essa população específica².

Partindo do princípio de que o cuidar do corpo humano exige, necessariamente, um olhar para a dimensão total do ser, inclusive de sua essência existencial daqueles que precisam de cuidados de enfermagem para se sentir mais 
Artigo Original

Atenção à Saúde

confortados, seguros, amparados, torna-se imprescindível, para nós enfermeiros, uma maior conscientização acerca do importante papel que desempenhamos ao interferir no espaço de privacidade das pessoas dependentes de nossas intervenções, como as pessoas com deficiências, sejam elas físicas ou intelectuais ${ }^{3}$.

A integralidade é uma palavra curiosa que não pode nem ao menos ser chamada de conceito. Na melhor das hipóteses, é uma rubrica conveniente para o agrupamento de um conjunto de tendências cognitivas e políticas com alguma imbricação entre si, mas não completamente articuladas. Pode-se identificar, grosso modo, um conjunto de tradições argumentativas que desembocam nesse agregado semântico: por um lado, um discurso propagado por organismos internacionais, ligado às ideias de atenção primária e de promoção de saúde; por outro, a própria demarcação de princípios identificada em pontos esparsos da documentação oficial das propostas de programas mais recentes do Ministério da Saúde em nosso país; por fim, nas críticas e proposições sobre a assistência à saúde de alguns autores acadêmicos em nosso meio. A inexistência de uma definição de fato sobre o que seria a tal 'integralidade' é ao mesmo tempo uma fragilidade e uma potencialidade, sendo ambas as características apontadas, ainda que nem sempre simultaneamente ${ }^{4}$.

Os cuidados específicos e gerais aos pacientes com necessidades especiais podem ser organizados e desempenhados pelo enfermeiro por meio da implantação e implementação da Sistematização da Assistência de Enfermagem (SAE), como uma metodologia de trabalho que orienta o cuidado profissional de Enfermagem.

As razões deste projeto vem da necessidade e da grande importância de um profissional enfermeiro na instituição da APAE para compor a equipe multiprofissional, sendo de extrema importância a presença deste profissional para a assistência ao cuidado e ao autocuidado destas pessoas portadoras de necessidades especiais, visando às necessidades da equipe multiprofissional relacionado ao cuidado de enfermagem na APAE de um município do Extremo Sul Catarinense, pois não existe equipe de enfermagem para o cuidado às crianças com deficiência intelectual.

Diante destas reflexões, surgiram alguns questionamentos relativos ao cuidado de enfermagem na APAE e as necessidades da equipe multidisciplinar, tais como: quais as características da população atendida na APAE? Quem são os profissionais que compõem a equipe? Quais os tipos de cuidados que essa população requer? Você está capacitado para atendê-los? Quais as principais dificuldades 
Artigo Original

Atenção à Saúde

vivenciadas no atendimento às intercorrências? Quando acontece algum problema de saúde a quem recorrem?

A partir destas inquietações, surgiu o problema de pesquisa frente à temática: Quais as necessidades da equipe multiprofissional relacionadas ao cuidado de enfermagem na APAE, de um município do Extremo Sul Catarinense?

Buscando identificar as necessidades da equipe multidisciplinar no cuidado em enfermagem elencou-se como hipóteses:

Não existe equipe de enfermagem para o cuidado às crianças com deficiência intelectual;

As necessidades da equipe multiprofissional da APAE frente ao cuidado de enfermagem estão relacionadas ao atendimento às crianças nas intercorrências clínicas relacionada às doenças e agravos existentes;

Existe possibilidade de inserção do profissional enfermeiro para o cuidado de enfermagem na APAE nas seguintes atribuições: educação em saúde com as crianças, pais e equipe multiprofissional; serviços de promoção e prevenção à saúde; assistência de enfermagem frente às necessidades apresentadas; encaminhamento dos casos quando necessário à rede de saúde; atendimento às intercorrências de urgência e emergência.

Com o intuito de conhecer as necessidades da equipe multiprofissional da APAE frente ao cuidado de enfermagem, objetivou-se identificar as necessidades da equipe multiprofissional da APAE relacionadas aos cuidados de enfermagem. Além disso, elencaram-se, a partir do objetivo geral, os objetivos específicos para identificar o perfil da Equipe Multiprofissional atuante na APAE: conhecer a capacitação dos profissionais para atuação nas possíveis intercorrências clínicas dos alunos; conhecer as principais dificuldades vivenciadas pela equipe multiprofissional relacionada ao atendimento das possíveis intercorrências clínicas dos alunos; identificar de que forma a equipe enfrenta estes desafios e conhecer as possibilidades de atuação relacionadas ao cuidado de enfermagem na APAE.

\section{MÉTODOS}

Pesquisa de abordagem qualitativa, descritiva, exploratória e de campo. $O$ estudo foi desenvolvido na APAE de um município do Extremo Sul de Santa Catarina. 
A análise de dados foi iniciada após a aprovação do Comitê de Ética em Pesquisa da instituição UNESC.

Foi aplicado um questionário semiestruturado com profissionais que compõe a equipe multiprofissional da instituição da APAE.

A escolha do questionário de maneira semiestruturado se deu como roteiro flexível visando obter respostas aos objetivos da pesquisa.

A análise dos dados foi realizada a partir da análise de conteúdo, a partir da categorização dos dados, através da ordenação, classificação e análise final dos dados pesquisados.

Para preservar o sigilo decorrente das entrevistas realizadas, de acordo com as diretrizes e normas regulamentadoras da Res. 466/12 (CNS) que envolvem pesquisa com Seres Humanos e Grupos Vulneráveis, utilizou-se a letra "P" para os profissionais da equipe multidisciplinar, seguida do respectivo número - P1 a P14.

A pesquisa foi aprovada pelo Comitê de Ética em Pesquisa da UNESC pelo Projeto no 1.466.438, iniciando-se a coleta de dados após a aprovação.

\section{RESULTADOS E DISCUSSÃO}

\section{Profissionais que compõe a equipe atuante na APAE}

A idade dos profissionais pesquisados variou de vinte e quatro (24) anos a sessenta e um (61) anos. Quanto ao gênero, todas do sexo feminino. O estado civil foram onze (11) casadas e três (3) solteiras. O tempo de trabalho dos profissionais na APAE esteve entre dois (2) meses a dezessete (17) anos. Quanto à formação destes profissionais estão: um (1) fisioterapeuta, um (1) psicólogo, um (1) professor de educação física, onze (11) pedagogos, sendo destes um (1) em andamento e três (3) com especialização em educação especial.

\section{Equipe de Enfermagem na APAE}

Todos os profissionais que responderam ao questionário relataram que não existe equipe de enfermagem atuante na APAE. 
A criança portadora de necessidades especiais sempre foi apontada como uma área especial na questão da assistência à saúde, tanto no Brasil como no resto do mundo. Esta constatação se faz pela simples observação desta preocupação na legislação atual, que, entre outros aspectos, procura garantir o acesso à saúde a todos os cidadãos e destaca a promoção de ações preventivas da deficiência, bem como programas especiais para prevenção de acidentes, reabilitação e habilitação junto a esta clientela.

Baseando-se nos aspectos legais da Enfermagem, é fundamental lembrar a lei n. 7.498/86, que dispõe sobre o exercício da enfermagem, na resolução do COFEN n. 146 de 1992, que dispõe sobre a normatização em âmbito nacional da obrigatoriedade de haver enfermeiro em todas as unidades de serviço, onde sejam desenvolvidas ações de enfermagem, durante todo o período de funcionamento da instituição de saúde 5 .

\section{A capacitação da Equipe Multiprofissional para atuar nas possíveis intercorrências clínicas dos alunos}

A capacitação da equipe multiprofissional da APAE se restringe às competências pedagógicas. A maioria dos professores referiu não se sentirem capacitados para agir frente às intercorrências clínicas, que são frequentes no cotidiano da instituição, conforme Quadro 1.

Quadro 1. Capacitação da equipe multiprofissional da APAE.

\begin{tabular}{|l|l|}
\hline \multicolumn{1}{|c|}{ Profissionais da APAE } & \multicolumn{1}{c|}{ № Professores (P) } \\
\hline $\begin{array}{l}\text { Formação profissional pedagogia e } \\
\text { como professor }\end{array}$ & $\mathbf{0 6}(\mathrm{P} 1 ; \mathrm{P} 5 ; \mathrm{P} 9 ; \mathrm{P} 10 ; \mathrm{P} 11 ; \mathrm{P} 12)$ \\
\hline Capacitação primeiros socorros & $\mathbf{0 3}$ (P6; P13; P14) \\
\hline Experiência como profissional & $\mathbf{0 3}$ P7; P8; P11; \\
\hline Nenhuma experiência & $\mathbf{0 2}(\mathrm{P} 2 ; \mathrm{P} 3)$ \\
\hline Experiência como mãe & $\mathbf{0 1}(\mathrm{P} 11)$ \\
\hline Não respondeu & $\mathbf{0 1}(\mathrm{P} 4)$ \\
\hline
\end{tabular}


Ressalta-se a importância de ter um profissional enfermeiro na equipe para o atendimento das intercorrências clínicas dos alunos, pois, conforme citado nos relatos, os profissionais que compõe o quadro atual da APAE não possuem qualificação para atuar nessas situações:

P1: "Pós-graduação em neuropsicopedagogia clínica."

P5: "Sou pedagoga."

P9: "A minha capacitação é na área pedagógica.".

P10: "Professora, noções de primeiros socorros."

P11: "Experiência como mãe e professora, tem que agir rapidamente e com coerência."

P12: "Pedagoga e especialista em Ed. Especial."

O perfil do educador deve ser crescentemente profissionalizado, no reconhecimento do saber advindo da experiência sem tornar-se um prático ativista, cuja atuação fica restrita ao imediatismo. Isso requer uma formação teórica sólida e crítica prévia e contínua ${ }^{5}$.

A APAE é uma instituição dentro de um contexto de socialização complementar à família, que deve propiciar cuidados de higiene, saúde, alimentação e educação, em um clima afetivo, estimulante e seguro às crianças, e um profissional enfermeiro dentro da equipe multidisciplinar, nesse sentido, pode atuar integralmente, avaliando a saúde da criança, referente ao crescimento e desenvolvimento infantil, com ações de prevenção e promoção da saúde a todos.

Como os profissionais da APAE não tem capacitação para agir nas intercorrências clínicas dos alunos, o risco de vida deles é maior, caso tivesse o profissional enfermeiro apto para avaliar as condições dos alunos e dar mais segurança aos mesmos.

A capacitação em primeiros socorros foi a base para o atendimento de intercorrências clínicas dos alunos da APAE, segundo os profissionais P6, P13 e P14. A leitura e estudo sobre o assunto (P6), além da formação profissional (P6 e P14) proporcionou capacitação para o atendimento destas intercorrências:

P6: "Minha formação: palestras sobre primeiros socorros, leituras." 
P13: "Primeiros socorros."

P14: "Como fisioterapeuta na verificação de sinais vitais, execução de algumas ações de primeiros socorros $e$ encaminhamentos quando necessário SAMU."

A experiência no âmbito profissional (P7, P8 e P11) e a experiência como mãe (P11) foi o alicerce para capacitar os profissionais nas possíveis intercorrências clínicas com os alunos da APAE. Os profissionais P2 e P3 não receberam nenhuma capacitação, somente a prática, e o profissional P4 não respondeu.

A educação permanente é compreendida como sendo um processo educativo contínuo, de revitalização e superação pessoal e profissional, de modo individual e coletivo, com objetivo de qualificação, reafirmação ou reformulação de valores, construindo relações integradoras entre os sujeitos envolvidos para uma praxe crítica e criadora ${ }^{6}$.

Com a presença do profissional enfermeiro atuante na equipe multidisciplinar na APAE, o mesmo poderia fazer educação em saúde com os próprios alunos, pais e equipe para uma melhor compreensão ou até mesmo uma capacitação para os mesmos.

\section{Condutas no atendimento às intercorrências clínicas}

Ter calma e tranquilidade são ideais. Segundo P2 e P3, quando não conseguem resolver, o aluno vai para casa. Porém, segundo P5 e P9, se não conseguem resolver em sala procuram auxílio aos demais profissionais da instituição.

As maiores dificuldades citadas pelos profissionais foram a agilidade para 0 atendimento e ainda a falta de autonomia e inexistência de protocolos para essas intercorrências vivenciadas pela equipe da APAE.

Para o profissional P11, não podem medicar, somente realizar os primeiros socorros, mas P14 ainda relata a dificuldade de assistência de kit adequado para realização dos primeiros socorros e o profissional capacitado para este atendimento.

A falta de informação e capacitação são as dificuldades relatadas pelo profissional P13, sendo que para P12 são as crises e os comportamentos dos alunos da instituição da APAE. 
Os profissionais P7 e P8 até o momento não tiveram nenhum caso e o profissional P1 ainda não teve esta experiência. O profissional P4 não teve casos de intercorrências, mas quando acontece algo com os alunos tem a equipe técnica como apoio, embora não conste o enfermeiro nessa equipe e os outros profissionais citados como apoio não cumpram esse papel.

\section{Característica da população atendida}

De acordo com os profissionais questionados na APAE, as características dos alunos atendidos (segundo P1, P4, P5, P6, P7, P8, P9, P12, P13 e P14) são deficiência intelectual moderada, múltipla e severa. $\mathrm{P} 6$ relata sobre a idade e ainda P7, P8 e P13 especificam algumas síndromes.

P1: "Alunos especiais, com deficiências múltiplas."

P4: "Alunos com deficiência intelectual e múltipla."

P5: "Pessoa com deficiência intelectual."

P6: "Pessoas de 0 anos sem limite de permanência, hoje o aluno mais velho tem 61 anos, pessoas com deficiência intelectual de moderada a severa e múltipla e síndromes associadas."

P7 e P8: "A APAE possui alunos com deficiência intelectual moderada à severa, múltiplas e síndromes associadas, tais como: Down, Aspeger, Rett, West, x-frágil, paralisia cerebral, hidrocefalia, microcefalia, Seckel entre outras."

P9: "Pessoa com deficiência intelectual."

P12: "Pessoa com deficiência intelectual e múltipla."

P13: "Autistas, Down, deficiência intelectual moderada, deficiências intelectuais, microcefalia, hidrocefalia e entre outras."

P14: "Pessoas com deficiência mental moderada e grave de etiologias diversas, com idade de 0 a 60 (média) anos. Alguns com deficiências físicas associadas ou outros comorbidades." 
A deficiência mental refere-se ao funcionamento intelectual geral significativamente abaixo da média, que coexiste com falhas no comportamento adaptador e se manifesta durante o período de desenvolvimento 7 .

\section{Os tipos de cuidado que os alunos da APAE necessitam}

A população atendida na APAE necessita de diversos cuidados diferenciados. Para os profissionais $\mathrm{P} 2, \mathrm{P} 3, \mathrm{P} 4, \mathrm{P} 5, \mathrm{P} 6, \mathrm{P} 9$ e P14 são realizadas atividades de vida diária de como se comportar em público, como se comunicar, como tomar banho, o cuidado pessoal, higiene e, o mais importante, a necessidade de dar amor, carinho e atenção.

Os tipos de cuidados que a população da APAE requer, para os profissionais P5, P7, P8 e P9, são a inclusão social e educacional, a ocupação, socialização e a comunicação dos alunos perante a sociedade. Muitas vezes, os próprios profissionais não visualizam a importância da enfermagem, devido à fragilidade dessas crianças que requer um cuidado mais acurado e especializado.

Além dos cuidados citados por outros profissionais, P1, P10 e P11 apresentam a verificação da pressão arterial (verificada pelo fisioterapeuta), a administração de medicações, realização dos primeiros socorros quando necessário e, para P13, o deslocamento do ambiente escolar, papel não condizente com suas funções, mas que pela ausência do profissional enfermeiro, acabam realizando e se expondo a riscos legais; P12 relata ser interventora e não cuidadora.

\section{Resolutividade da intervenção nas intercorrências clínicas}

De acordo com a gravidade do caso da intercorrência clínica e para uma melhor segurança aos alunos da instituição, os profissionais realizam os primeiros atendimentos e primeiros socorros (P1, P4 e P13); na falta de resolutividade, resolvem ligam para família e em seguida para o SAMU (P4, P6, P10, P11, P12, P13 e P14).

O profissional $\mathrm{P9}$ prefere resolver a intercorrência, caso não consiga, procura uma assistência como os outros profissionais, como ressaltam P5, P7, P8, P9 e P14, acreditando que seja o melhor a fazer.

Quando ocorre uma intercorrência clínica na APAE os profissionais P2 e P3 recorrem somente aos pais. 


\section{Necessidades da equipe multiprofissional: Opinião da Equipe multiprofissional da APAE sobre a inclusão do Enfermeiro na equipe dos profissionais da APAE}

Todos os profissionais consideram importantes e necessários a incorporação do Enfermeiro na equipe multiprofissional, pois proporcionaria maior segurança, eficácia e qualidade na assistência prestada, possibilitando melhor desenvolvimento e qualidade de vida ao aluno da APAE:

P1: "Penso que será muito bom, mais segurança e eficácia para o aluno e escola."

P2: "É muito importante e necessário."

P3: "É ótima, pois iria ajudar muito."

P4: "Será de grande ajuda, pois ficaríamos mais seguros."

P5: "Muito bom."

P6: "Extremamente necessário, somaria na equipe e melhoraria 0 atendimento ao aluno."

P7 e P8: "É de extrema importância para a preservação e manutenção da saúde dos alunos. Sempre pensando no bem estar de cada aluno."

P9: "Precisamos de um enfermeiro na APAE."

P13: "Excelente."

P14: "Muito importante. Beneficiaria os alunos e demais profissionais da equipe."

De acordo com a lei do exercício profissional Lei ํㅜ 7.498/86 o profissional enfermeiro exerce todas as atividades de enfermagem, sendo que privativamente atua na direção e avaliação dos serviços e assistência de enfermagem; consulta de enfermagem; assistência de enfermagem de maior complexidade técnica e cuidados diretos a pacientes graves com risco de vida ${ }^{1}$.

Devido à ausência do profissional enfermeiro na instituição as ações privativas do enfermeiro, conforme a lei, são executadas por outros profissionais da equipe que 
Artigo Original

Atenção à Saúde

não estão aptos e correm risco com o desvio de funções, conforme protocolos de cada profissional.

Com a inserção do profissional enfermeiro na equipe multidisciplinar da APAE o mesmo poderá fazer todas as funções que lhe cabe, não somente procedimentos, incluindo as educações em saúde, promovendo a promoção e prevenção de saúde para os alunos, pais e até mesmo com os professores.

\section{As necessidades da equipe multiprofissional da APAE relacionado ao cuidado de enfermagem}

Para os profissionais P1, P2, P3 e P14 ter um profissional enfermeiro é uma necessidade da equipe multiprofissional na APAE, melhoraria a qualidade de assistência à saúde dos alunos, sendo de grande importância este profissional na equipe multidisciplinar para sanar as dúvidas. Ainda (segundo P1), seria importante ter uma sala apropriada para um melhor atendimento.

A necessidade da inserção do enfermeiro na equipe, para os profissionais $\mathrm{P} 4$, P5, P6, P7, P8, P12 e P13, se traduz na realização de alguns cuidados, como: verificação de pressão arterial, administração de medicamentos, curativos em casos de crises e intercorrências cotidianas, reconhecendo as especificidades de cada profissão e o núcleo de saber de cada um.

O método de trabalho que a enfermagem lança mão para realizar as suas atividades de maneira científica denomina-se Sistematização da Assistência de Enfermagem (SAE). A construção do conhecimento em enfermagem e as vivências têm início ainda na graduação e, quando se encontram em consonância com a filosofia do serviço, favorecem práticas qualificadas ao paciente, propiciando um atendimento integral as necessidades humanas básicas².

Em uma instituição de educação especial, a exemplo da APAE, podem-se encontrar pessoas com rebaixamento intelectual, transtornos físicos e mentais associados e situações clínicas com características próprias e singulares².

A Política Nacional de Saúde da Pessoa Portadora de Deficiência pauta-se no pressuposto de que além da atenção à saúde específica da sua condição, este é um cidadão que pode ser acometido por doenças e agravos comuns aos demais. 


\section{Atenção à Saúde}

Portanto, faz-se necessário dispor de uma equipe multiprofissional que tenha como pressuposto a visão holística do ser humano ${ }^{8}$.

Neste sentido, a enfermagem pode desenvolver um importante papel na promoção da saúde desta população. Por meio da educação, constroem-se cidadãos e a educação em saúde é uma das alternativas que a enfermagem dispõe para auxiliar estas pessoas a tornarem-se indivíduos ativos na construção de sua vida e de sua independência. A enfermagem, no contexto de educar, pode atuar na educação especial promovendo o desenvolvimento das potencialidades das pessoas com deficiência.

As necessidades da equipe multiprofissional da APAE relacionadas ao cuidado de enfermagem são apresentadas, para P9, P10 e P11, pelo relato de que cada profissional tem suas responsabilidades, e na ausência do enfermeiro não há a atuação e o cuidado do mesmo.

\section{Possibilidades de atuação do enfermeiro na APAE}

A complexidade das necessidades demandadas pelas pessoas que frequentam a APAE e a responsabilidade que recai sobre a equipe sem preparo para o enfrentamento das situações que se apresentam fica visível quando olhamos o Quadro 2 e observamos as falas ali contidas:

Quadro 2. Possibilidades de atuação do enfermeiro na APAE.

\begin{tabular}{|l|l|}
\hline $\begin{array}{l}\text { Possibilidades de atuação do } \\
\text { enfermeiro }\end{array}$ & No Professores (P) \\
\hline $\begin{array}{l}\text { Atendimento às intercorrências de } \\
\text { Urgência e Emergência }\end{array}$ & $\begin{array}{l}14 \text { (P1; P2; P3; P4; P5; P6; P7; P8; } \\
\text { P9; P10; P11; P12; P13; P14) }\end{array}$ \\
\hline $\begin{array}{l}\text { Assistência de Enfermagem frente às } \\
\text { necessidades apresentadas }\end{array}$ & $\begin{array}{l}13 \text { (P2; P3; P4; P5; P6; P7; P8; P9; } \\
\text { P10; P11; P12; P13; P14) }\end{array}$ \\
\hline $\begin{array}{l}\text { Encaminhamento dos casos quando } \\
\text { necessário a rede de saúde }\end{array}$ & $\begin{array}{l}\mathbf{1 3} \text { (P1; P2; P3; P4; P5; P6; P7; P8; } \\
\text { P9; P10; P11; P13; P14) }\end{array}$ \\
\hline Educação em saúde & $\begin{array}{l}\mathbf{1 2} \text { (P1; P2; P3; P4; P5; P6; P7; P8; } \\
\text { P9; P10; P11; P14) }\end{array}$ \\
\hline
\end{tabular}


Artigo Original

Atenção à Saúde

\begin{tabular}{|l|l|}
\hline $\begin{array}{l}\text { Possibilidades de atuação do } \\
\text { enfermeiro }\end{array}$ & No Professores (P) \\
\hline Promoção e Prevenção à saúde & $\begin{array}{l}12 \text { (P2; P3; P4; P5; P6; P7; P8; P9; } \\
\text { P10; P11; P13; P14) }\end{array}$ \\
\hline Outros & $\mathbf{0 3 ~ ( P 6 ; ~ P 9 ; ~ P 1 0 ) ~}$ \\
\hline
\end{tabular}

A inclusão de enfermeiros se faz necessária na instituição da APAE, considerando que seus cursos de formação conferem qualificação para o cuidado integral da criança, desde a sua concepção. Os cursos de graduação capacitam o profissional para atuar nas ações primárias e assistindo a criança e sua família na promoção e prevenção de saúde 5 .

A enfermagem, juntamente com outros membros, é capaz de desenvolver um importante papel na promoção do autocuidado, tendo como função modificar o ambiente para aprendizagem e gerar comportamentos mais independentes, ajudando os portadores de deficiências a desenvolver seus talentos ao máximo e remover, quando possível, o preconceito que existe em relação aos portadores de necessidades especiais.

\section{Intercorrências e problemas de saúde: a quem recorrem}

Quando acontece alguma intercorrência ou até mesmo um problema de saúde com os alunos da APAE, chamam a família (P11 e P12) em casos que não conseguem resolver na instituição e os casos mais graves acionam o SAMU (P5, P6, P9, P11, $\mathrm{P} 12, \mathrm{P} 13$ e P14), e outras levam até um pronto-atendimento ou até mesmo unidade hospitalar (P6 e P14).

A assistente social, para P2, P3, P4 e P10, é a acionada em caso de intercorrências ou problemas de saúde com os alunos da APAE e, ainda P2 e P3 fazem ligação para família. Para os profissionais P1, P7, P8 e P13, quando acontece algum problema de saúde recorrem às orientadoras da escola e, ainda $\mathrm{P} 13$, se for mais grave, é acionado o Serviço de Atendimento Móvel de Urgência (SAMU).

O SAMU destina-se ao atendimento de urgência e emergência nas residências, locais de trabalho e vias públicas. O socorro é feito depois de chamada gratuita para o telefone 192. A ligação é atendida por técnicos na central de regulação 
que imediatamente transferem o telefonema para o médico regulador. Esse profissional faz o diagnóstico da situação e inicia o atendimento no mesmo instante, orientando o paciente ou a pessoa que fez a chamada sobre as primeiras ações ${ }^{9}$.

Com a presença do profissional enfermeiro na equipe multidisciplinar da APAE, o mesmo já pode realizar os primeiros cuidados para estabilização do quadro de urgência ou emergência, e o paciente estaria pronto para ser levado pelo SAMU na chegada, facilitando e adiantando toda a situação e ganhando mais tempo para salvar a vida do aluno.

\section{Sugestões sobre a temática: $O$ cuidado de enfermagem na APAE: necessidades da equipe multiprofissional}

Como forma de sugestão sobre a temática dada pela equipe da APAE, surgiu de forma bastante enfática a necessidade da contratação de um profissional enfermeiro na instituição (de acordo com P1, P2, P3, P4, P7, P8, P10, P11, P12 e $\mathrm{P} 13$ ), uma sala equipada para o mesmo poder atuar (de acordo com P1), onde teria uma grande importância e uma melhora no quadro da equipe multiprofissional da APAE, que resultaria em uma melhor qualidade de vida aos alunos da instituição.

P1: "A contratação de um enfermeiro efetivo e uma sala equipada".

P2: "Que seria muito importante e de fundamental necessidade um profissional enfermeiro."

P3: "Uma enfermeira ajudará muito na APAE."

P4: "Cursos. Uma enfermeira na escola todos os dias ou algumas vezes por semana."

P7: "É uma proposta muito interessante, o trabalho de um enfermeiro junto à equipe de profissionais da APAE resultaria em uma melhor qualidade de vida aos nossos alunos."

P10: "Sugerimos o trabalho de enfermagem para ajudar a melhorar a saúde dos alunos e a melhora dos cuidados." 
P11: "A escola, pelo número elevado de alunos, e pelas situações diferenciadas que ocorrem, pois cada aluno requer cuidados. Sugerimos o serviço de enfermagem".

P12: "Pelas limitações da clientela escolar, seria importante um profissional na área de enfermagem na instituição."

P13: "Será de grande valia ter uma enfermeira na equipe de profissionais da APAE."

\section{CONCLUSÕES}

A Política Nacional de Saúde da Pessoa Portadora de Deficiência pauta-se no pressuposto de que além da atenção à saúde específica da sua condição, este é um cidadão que pode ser acometido por doenças e agravos comuns aos demais. Portanto, faz-se necessário dispor de uma equipe multiprofissional que tenha como pressuposto a visão holística do ser humano.

Neste sentido, a enfermagem pode desenvolver um importante papel na promoção da saúde desta população. Por meio da educação constroem-se cidadãos, e a educação em saúde é uma das alternativas que a enfermagem dispõe para auxiliar estas pessoas a tornarem-se indivíduos ativos na construção de sua vida e de sua independência. A enfermagem, no contexto de educar, pode atuar na educação especial.

Como resultado da pesquisa foi possível identificar o perfil da equipe multiprofissional atuante e suas capacitações, na qual não estão aptos para proporcionar uma melhor assistência e qualidade na vida e na saúde do aluno, sendo que se tivesse o profissional enfermeiro atuante na APAE teria melhores condições na saúde do aluno.

Uma das vantagens que o enfermeiro proporcionaria na APAE em relação à saúde dos alunos seriam orientações específicas, maior tranquilidade e segurança para os familiares, mais um profissional de saúde que se agrega aos outros profissionais já existentes para a melhoria do atendimento aos alunos, uma possível diminuição no número de intercorrências, proporcionando assim uma melhor qualidade de vida em relação à saúde dos alunos da instituição. 
Artigo Original

Atenção à Saúde

Acreditamos que a presença do enfermeiro nestas instituições pode contribuir para a diminuição do sofrimento, bem como para a melhoria da qualidade de vida das pessoas com variados tipos de deficiência e necessidades de saúde.

\section{REFERÊNCIAS}

1. Brasil. Dispõe sobre a regulamentação do exercício da Enfermagem e dá outras providências. [internet]. Lei no 7.498/86. [acesso em 2016 fev 15]. Disponível em: http://www.abennacional.org.br/download/leiprofissional.pdf

2. Adamy E, Krauzer I, Hillesheim C, Silva B, Garghetti F. A inserção da sistematização da assistência de enfermagem no contexto de pessoas com necessidades especiais. Rev. Pesqui. Cuid. Fundam. 2013;5(3):53-65.

3. Silva KL, Sena RR. Integralidade do cuidado na saúde: indicações a partir da formação do enfermeiro. Rev. Esc. Enferm. USP, São Paulo. 2008;42(1):48-56.

4. Pinheiro R, Mattos RA. Os sentidos da integralidade na atenção e no cuidado à saúde. (Org). Rio de Janeiro: UERJ, IMS: Abrasco, 2006.

5. Viana ACR, Santos TMS, Sandos HFL, Moraes KF, Brasileiro ME. A importância da atuação do enfermeiro na assistência a crianças em creches municipais de Goiânia. Rev.Eletrônica Enfer. 2009;1(3):1-17.

6. Tavares CMM. A educação permanente da equipe de enfermagem para o cuidado nos serviços de saúde mental. Texto \& Contexto Enfermagem. 2006;15(2):287-95.

7. Kirk SA, Gallagher JJ. Educação da criança excepcional. 3.ed. São Paulo: Martins Fontes, 1996. 502p.

8 Brasil. Ministério da Saúde. Secretaria de Atenção à Saúde. Política Nacional de Saúde da Pessoa Portadora de Deficiência. Brasília (DF): Ministério da Saúde, 2008.

9. Cabral APS, Souza WV. Serviço de Atendimento Móvel de Urgência (SAMU): análise da demanda e sua distribuição espacial em uma cidade do Nordeste brasileiro. Rev Bras Epidemiol. 2008;11(4):530-40. 